\title{
A PSICOLOGIA NA PRODUÇÃO CIENTÍFICA NACIONAL DE ENFERMAGEM
}

\author{
Ana Maria Pimenta Carvalho* \\ Graziela Valentina Pavan de Arruda Camargo**
}

Carvalho AMP, Camargo GVPA. A psicologia na produção científica nacional de enfermagem. Rev Latino-am Enfermagem 2001 março; 9(2): 61-6.

O presente trabalho buscou, através de consultas a periódicos da área de Enfermagem, identificar elementos de interação desta com a Psicologia. Foram selecionados 317 artigos que enquadraram-se no critério de incluir em sua abordagem questões psicológicas e sua análise compreendeu: 1. a temática focalizada; 2. a categoria profissional dos autores; 3. as referências a obras de Psicolologia; 4. modelos teóricos em Psicologia e 5. metodologia. Concluiu-se que a forma como essa interação vem operando, no contexto focalizado, é através do diálogo teórico-metodológico. 0 contato entre profissionais de diferentes formações apareceu em pequena proporção.

UNITERMOS: psicologia, enfermagem, produção científica

\section{PSYCHOLOGY IN NATIONAL NURSING SCIENTIFIC PUBLICATIONS}

This study aimed, through a review of Nursing periodicals, at identifying elements of interaction between Nursing and Psychology. Authors selected 317 articles that met the criterion of approaching psychological issues. The analysis included: 1. the focussed theme; 2.authors' position; 3. references to Psychology works; 4. Psychology's theoretical frameworks and 5. methodology. Authors concluded that this interaction is occurring through a theoretical-methodological dialogue. The contacts among professionals with different academic backgrounds were scarce.

KEY WORDS: psychology, nursing, scientific publications

\section{LA PSICOLOGÍA EN LA PRODUCCIÓN CIENTÍFICA NACIONAL DE ENFERMERÍA}

El presente trabajo buscó identificar, en revistas de Enfermería, los elementos de interacción de ésta con la Psicología. Fueron seleccionados 317 artículos que se encuadraron en el criterio de incluir en su enfoque, cuestiones psicológicas y su análisis comprendió: 1. la temática enfocada: 2. la categoría profesional de los autores; 3. las referencias a las obras de Psicología; 4. Modelos teóricos de Psicología y 5. la metodología. Se concluyó que la forma como esa interacción ocurre en el contexto enfocado, es a través del diálogo teórico-metodológico. El contacto entre profesionales de diferentes formaciones apareció en pequeña proporción.

TÉRMINOS CLAVES: psicología, enfermería, producción científica

\section{INTRODUÇÃO}

$\boldsymbol{E}_{\text {ste trabalho foi idealizado a partir da necessidade de }}$ se conhecer como vem ocorrendo a inserção do conhecimento da área da Psicologia, na Enfermagem. Optou-se por apreendê-la a partir da produção científica nacional de Enfermagem. Infere-se que a incursão pelos trabalhos publicados permitirá uma compreensão da interação entre as duas, uma vez que enquanto disciplina a Psicologia faz parte dos cursos de formação do enfermeiro, médico e profissionais da Saúde. Esta interação é de tal modo estreita que

\footnotetext{
* Psicóloga, Docente do Departamento de Enfermagem Psiquiátrica e Ciências Humanas da Escola de Enfermagem de Ribeirão Preto da Universidade de São Paulo, Centro Colaborador da Organização Mundial da Saúde para o desenvolvimento da pesquisa em Enfermagem. Endereço: Av. Bandeirantes, 3900 - Monte Alegre - 14040-902 - Ribeirão Preto - São Paulo - Brasil. E-mail: mcsouza@highnet.com.br

** Aluna do $7^{\circ}$ semestre do Curso de Graduação em Enfermagem da Escola de Enfermagem de Ribeirão Preto da Universidade de São Paulo
} 
a sub área da Psicologia ${ }^{(1)}$, relativa ao estudo da Personalidade, nasceu no contexto de uma prática médica que buscou na Filosofia ampliar seus conceitos sobre o funcionamento humano.

Ainda, a relação entre Psicologia e a área da Saúde refletese, no início do século XX, com a criação da disciplina chamada Psicologia Médica, por Kretschmer que, na década de 60 passou a fazer parte dos curricula de cursos de Medicina, no Brasil ${ }^{(2)}$.

No currículo de Enfermagem, ao lado das Ciências Biológicas, as Ciências Humanas devem incorporar-se ao "fazer/ pensar do enfermeiro" ${ }^{(3)}$. Dentre elas, a Psicologia foi inserida no currículo de cursos de Enfermagem a partir do decreto/lei 775, datado de 1949, quando estes ainda eram cursos de nível médio. No final da década de 50 houve sua proposição como curso de nível universitário, o que passou a ser efetivado no início da década de $60^{(4)}$.

Foi a partir da década de 50 que, mais sistematicamente, a área da Saúde começou a empreender esforços no sentido de compreender as questões a ela concernentes não apenas no contexto do funcionamento biológico do organismo humano. Para tanto, recorreu às Ciências Humanas para assim integrar um conhecimento do homem que fosse mais totalizador ${ }^{(5)}$.

A adoção do conceito de saúde entendido como uma qualidade inerente ao homem, resultante do consumo de uma série de meios de vida (entre os quais a Medicina e a Psicologia, através da prestação de serviços, são apenas alguns aspectos) e da capacidade produtiva do indivíduo vêm exigindo uma leitura plural do fenômeno saúde-doença" ${ }^{(6)}$.

0 fenômeno saúde-doença é também o objeto da atenção da Enfermagem que se caracteriza pela atuação na assistência direta ou indiretamente ao indivíduo que adoece, na prevenção de agravos, na promoção da saúde, planejando estratégias eficazes para 0 alcance de seu bem estar físico e mental e pela busca da compreensão do processo de sua construção, não somente no plano individual, mas social.

Alguns autores analisaram a produção da Enfermagem no âmbito da Iniciação Científica, no período de 1993 a 1996, veiculada nos anais de dois eventos, o Simpósio de Iniciação Científica da Escola de Enfermagem de Ribeirão Preto e o Simpósio de Iniciação Científica na Universidade de São Paulo ${ }^{(7)}$. Encontraram que a maior parte dos trabalhos abordava aspectos sócio culturais envolvidos no trabalho do enfermeiro. Como segunda categoria mais freqüente apareceu a abordagem a aspectos biológicos. Os aspectos psicológicos apareceram em proporções bem inferiores, às outras duas categorias. As autoras argumentam que este achado se deveu ao fato de que, na pós-graduação, as disciplinas relacionadas às Ciências Sociais ocupavam uma proporção significativa do total de disciplinas oferecidas e, visto que os docentes responsáveis por elas eram também os orientadores dos trabalhos de Iniciação Científica, explicava-se as altas proporções de ocorrência de trabalhos inseridos nessa área.

Esse trabalho permite uma visão da interação da Enfermagem com as Ciências Sociais, especialmente no âmbito da pesquisa.

Tendo como meta descrever a presença da Psicologia no conhecimento produzido e publicado da Enfermagem. buscou-se, no presente trabalho, responder às seguintes questões:

Em que contexto de atuação o enfermeiro aborda os aspectos psicológicos da pessoa? Entende-se por psicológico o comportamento, os processos mentais e as relações indivíduo ambiente $^{(8-9)}$.

Há participação de profissionais psicólogos (docentes e técnicos) na autoria dos trabalhos?

São utilizadas, nos trabalhos analisados, referências a obras de Psicologia?

Qual a fundamentação teórica utilizada?

Qual a metodologia utilizada?

\section{MÉTODO}

Para compor o conjunto de trabalhos a serem analisados, procedeu-se a um levantamento junto a sete periódicos de Enfermagem, editados em diferentes estados do país, buscando, assim, cobrir uma gama representativa das publicações na área e que estivessem disponíveis na Biblioteca Central do Campus da Universidade de São Paulo, em Ribeirão Preto.

Foram selecionados os seguintes periódicos: Revista Baiana de Enfermagem, Revista Brasileira de Enfermagem, Revista da Escola de Enfermagem da USP de São Paulo, Revista da Escola de Enfermagem da Universidade Estadual do Rio de Janeiro, Revista Gaúcha de Enfermagem, Revista Latino Americana de Enfermagem e Enfermagem: Texto e Contexto, cobrindo o período de 1985 a 1997, exceto para as Revistas da UERJ e Latino-Americana que iniciaram suas edições em 1993 e para a Revista Baiana que interrompeu suas edições nos anos de 1989, 1990 e 1991.

Optou-se por um levantamento que se pode chamar de manual, visto não envolver tecnologias como a informática. As revistas foram consultadas uma a uma.

0 critério de inclusão dos trabalhos, no conjunto a ser analisado, foi ter mencionado nos unitermos ou palavras-chave os vocábulos Psicologia ou Psicológico ou aqueles relativos a processos psicológicos tais como Percepção, Comunicação, Relação Interpessoal. Um dos trabalhos, a título de exemplo, assim se apresentava: título do trabalho "Estudo sobre crianças queimadas: 
uma proposta de assistência em enfermagem" Unitermos Aspectos Psicológicos.

Entretanto, nem sempre as revistas adotavam a exibição de unitermos ou palavras-chave, por isso foi necessário ler os artigos e buscar neles o que poderiam trazer de psicológico. Em seguida, procedia-se à seleção a partir da leitura e registrava-se em um formulário as seguintes informações sobre os trabalhos: 1. autoria e qualificação profissional do primeiro autor; 2. informações sobre a data da publicação e o veículo; 3. foco de atuação profissional, presente no trabalho; 5 . estratégias metodológicas utilizadas e 5. referências a trabalhos da área da Psicologia.

A seleção foi revisada pelas pesquisadoras. Quando necessário procedia-se à releitura do artigo para manter sua inclusão ou não.

0 acordo entre as pesquisadoras, quanto à classificação dos artigos, atingiu um índice de aproximadamente $80 \%$.

A seleção dos trabalhos levou à obtenção de um conjunto de 317 artigos cuja distribuição, nos periódicos consultados, pode ser vista no Tabela 1.

\section{Tabela 1 - Número de artigos consultados e selecionados em cada periódico}

REUISTR/PERIÓOICO ABTIGOS ARTIGOS PORCENTAGEM

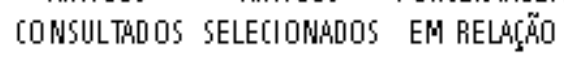
AO TOTRLL DE ARTIGOS CONSULTRDOS

$\begin{array}{cccc}\text { Baiana }^{\prime} & 110 & 22 & 20,0 \\ \text { Brasileira de Enf. }^{2} & 463 & 92 & 19,9 \\ \text { Escola de Enf. da UERI] }^{3} & 76 & 16 & 21,1 \\ \text { Escola de Enf./USP/SP }^{4} & 338 & 88 & 26,0 \\ \text { Gaúcha }^{3} & 213 & 50 & 23,5 \\ \text { Latino-Ámericana }^{6} & 160 & 24 & 15,0 \\ \text { Texto e Contexto }^{7} & 188 & 25 & 13,3 \\ \text { Total } & 1548 & 317 & 20,5\end{array}$

${ }^{1}$ Não foi consultado o $\mathrm{n}^{0} 2$ de 1988, por não estar disponível. E nos anos de 1989, 1990 e 1991 a revista não foi editada

${ }^{2}$ Não foram consultados os números 4 de 1986 e 2 de 1997 por não estarem disponíveis nas bibliotecas

${ }^{3}$ Os números de 1997 não estavam disponíveis. A revista iniciou suas atividades em 1997

${ }^{4}$ Não foram consultados os números 2 de 1989 e o 2 e 3 de 1994 por não estarem disponíveis. Os números de 1995 também não estavam disponíveis

${ }^{5}$ Não foram consultados os números de 1997 por não estarem disponíveis

${ }^{6}$ A revista iniciou suas atividades em 1993

${ }^{7}$ Não foram consultados os números 1 de 1992 e 2 de 1994 por não estarem disponíveis. A revista começou suas atividades em 1992

Verifica-se uma distribuição que varia de $13 \%$ a $26 \%$ na proporção de artigos que tratam de questões psicológicas. As revistas que menos abordam esses aspectos são a Texto e Contexto e a Latino-Americana. Para as demais revistas, a proporção varia de $20 \%$ a $23 \%$. Pode-se dizer que são proporções semelhantes. A Revista da EEUSP/SP é a que tem a maior proporção de artigos focalizando aspectos psicológicos.

Dentro dos itens que foram propostos para verificação da presença da Psicologia, a partir de sua ocorrência em trabalhos de Enfermagem, focalizou-se:

1. Categorias de atuação do Enfermeiro - buscou-se, neste sentido, identificar campos de inserção do trabalho do Enfermeiro que exigiam a interação entre as disciplinas/ áreas aqui focalizadas.

2. Autoria dos trabalhos - buscou-se identificar se havia, como primeiro autor, a presença de profissionais da área de Psicologia.

3. Referência a obras de Psicologia - buscou-se identificar sua presença nas listas de referência bibliográficas dos trabalhos.

4. Fundamentação teórica - buscou-se identificar qual a fundamentação teórica, em Psicologia, adotada no trabalho, considerando-se as seguintes linhas: psicodinâmica, fenomenológica, comportamental e humanista.

Calcularam-se as proporções, em que cada modelo teórico foi citado nos trabalhos, tomando-se cada ocorrência de uma obra, que se enquadrava num deles, sobre o total de artigos em que havia citação de referência de obras da área da Psicologia. Quando havia, por exemplo, a citação, num mesmo trabalho, de mais de uma obra de Carl Rogers, apenas uma ocorrência era registrada para o cálculo da proporção.

A identificação das referências foi feita considerando-se, no rol das referências, a ocorrência de uma obra de determinado autor. Assim, mesmo que houvesse a citação de três trabalhos de Carl Rogers, por exemplo, apenas uma referência à abordagem humanista/ centrada na pessoa era registrada.

5. Metodologia utilizada - buscou-se identificar que estratégias metodológicas eram utilizados nos estudos empíricos.

\section{RESULTADOS}

Neste trabalho os resultados serão tratados considerandose o total de artigos encontrados no conjunto dos sete periódicos focalizados.

Em relação ao contexto de atuação dos enfermeiros, foram identificadas as seguintes categorias:

1a. Assistência ao paciente/cliente (AP) - Refere-se a trabalhos que abordam 0 atendimento global ao paciente/clientes, por exemplo, atuações junto ao paciente colostomizado, deficiente mental, diabético, pacientes submetidos a cirurgias cardíacas e assistência a escolares, entre outros.

1b. Assistência a familiares de pacientes/clientes (AF) - Refere-se a 
trabalhos que abordam o atendimento dispensado a pessoas da família, por exemplo, trabalho com pacientes portadores de problemas ortopédicos em casa, cuidados com o paciente diabético, entre outros.

2a. Reações emocionais do paciente/cliente (REP) - Refere-se a trabalhos que colocam ênfase na questão emocional destacando-a, por exemplo, reações emocionais de portadores de DST; reações de mulheres frente a mastectomia, reações de pacientes paraplégicos frente a alterações nas funções sexuais, entre outros. 2b. Reações emocionais de familiares (REF) - Refere-se a trabalhos que enfatizam o envolvimento emocional dos familiares dos pacientes/clientes, por exemplo, necessidades emocionais de esposas de pacientes infartados, vivência de pais em relação à doença do filho, entre outros.

3. Reflexões sobre e/ou análise da profissão de enfermeiro e de sua prática (AP) - Refere-se a trabalhos que se centram na análise da prática profissional e nas concepções que se têm dela, por exemplo, significado do cuidar de pacientes com AIDS, percepção de alunos de graduação do papel de enfermeiro, enfermagem em UTI, ansiedade dos profissionais no contexto hospitalar, entre outros. 4. Ensino de Enfermagem na Graduação (EG) - Refere-se a trabalhos que abordam aspectos do ensino e da formação do profissional de Enfermagem, por exemplo, formação do aluno para atuar em programas de assistência à criança, o uso de psicodrama na sala de aula, ensino do exame físico, entre outros.

5. Interação/comunicação enfermeiro paciente (IEP) - Refere-se a trabalhos que têm como objetivo a descrição e análise da interação enfermeiro paciente, por exemplo, relação terapêutica enfermeiro psiquiátrico paciente; comunicação enfermeiro paciente no hospital. 6. Enfermeiro na equipe multiprofissional (EM)-Refere-se a trabalhos que descrevem a inserção do enfermeiro numa equipe, por exemplo, atendimento multiprofissional a programa de laqueadura; atendimento em equipe multiprofissional a pacientes diabéticos.

7. Outros (Ots.)- Refere-se a trabalhos que não puderam ser agrupados nas categorias anteriores, por exemplo, relato do próprio paciente sobre internação em UTI, rótulos em psiquiatria, interdisciplinaridade e saúde mental.

A quantificação relativa à identificação dessas categorias nos artigos revisados pode ser vista na Figura 1. A legenda indica as siqlas das cateqorias listadas acima.

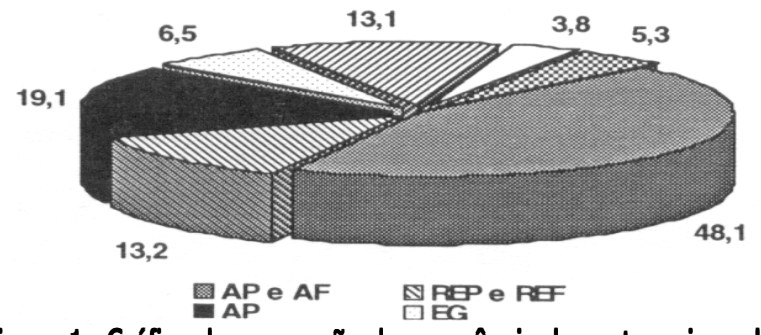

Figura 1 - Gráfico da proporção de ocorrência das categorias relativas ao contexto de atuação do enfermeiro, em que foi requerida a abordagem a aspectos psicológicos, Ribeirão Preto 1999
De modo geral, é na assistência ao paciente ou a familiares que emerge a necessidade de se abordar os chamados aspectos psicológicos. Esta categoria de trabalhos envolve uma assistência global, focalizando tanto aspectos físicos como emocionais e comportamentais. Segue-se a categoria de trabalho que engloba as reflexões/análise da prática profissional, especialmente focalizando aspectos relacionados ao sofrimento do enfermeiro, aos modos como ele lida com a tensão que o exercício da profissão the impõe. Como terceira categoria mais freqüente, verificam-se tanto aquela que engloba trabalhos relacionados às reações emocionais dos clientes e seus familiares como a que focaliza a interação enfermeiro paciente. As categorias menos freqüentes são as relativas ao ensino na graduação e atuação do enfermeiro em equipe multiprofissional.

Interessou, ainda, neste trabalho, identificar a categoria funcional dos primeiros autores dos artigos (Figura 2).

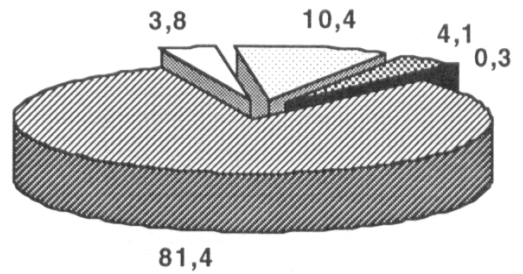
$\begin{array}{ll}\text { docente enfermeiro } & \square \text { docente não enfermeiro } \\ \text { enfermeiro de campópo } & \text { aluno de graduação } \\ \text { docente ensino médio } & \end{array}$

Figura 2 - Gráfico da proporção de ocorrência das diferentes categorias profissionais, como primeiro autor, nos artigos analisados, Ribeirão Preto, 1999

Como seria esperado, a grande maioria dos autores é enfermeiro docente universitário. A segunda categoria de autores, mais freqüente, é a de enfermeiros de campo, seguida de alunos de graduação.

Uma categoria não incluída aqui, mas que apareceu em alguns trabalhos, foi a de pós graduandos. Entretanto, como havia sobreposição com as categorias de docentes e enfermeiros de campo, optou-se por sua exclusão. Docentes não enfermeiros (médicos, filósofos, pedagogos, psicólogos) participaram, também, da autoria dos trabalhos, como primeiro autor.

Os trabalhos foram analisados, ainda, quanto a trazerem ou não em suas referências bibliográficas obras de Psicologia.

Os resultados deste levantamento, em termos de proporções, podem ser vistos na Figura 3.

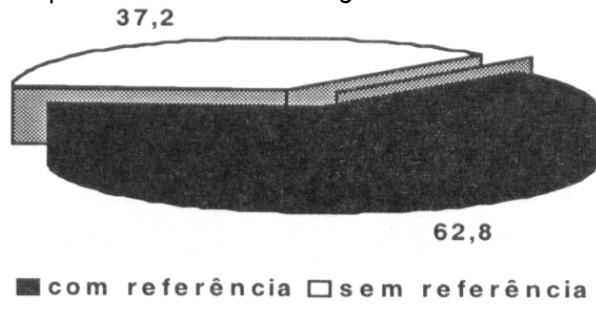

Figura 3 - Gráfico da proporção de artigos que apresentaram ou não referências a trabalhos de Psicologia, Ribeirão Preto, 1999 
A maior parte dos artigos selecionados faz referências a obras da área de Psicologia e esta porção abarca as sub áreas de Personalidade, Desenvolvimento e Saúde, 53,8\%. Seguem-se as áreas de Psiquiatria/Psicopatologia, que fazem interface com a Psicologia, 6,2\% e a Psicologia da Educação, 2,8\%. Embora as referências predominem, há uma proporção significativa de artigos, nos quais abordam-se os aspectos psicológicos, sem referências a trabalhos de Psicologia, mas a trabalhos da própria Enfermagem sobre os temas focalizados.

Ao fazer referência a modelos teóricos em Psicologia verificam-se os seguintes resultados proporcionais, exibidos na Figura 4.

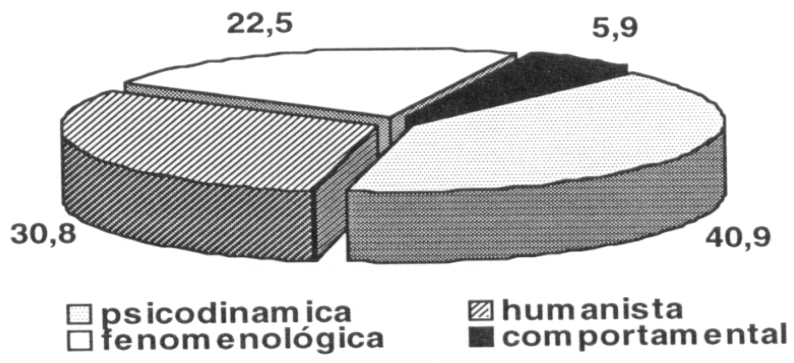

Figura 4 - Gráfico das referências aos diferentes modelos teóricos, em Psicologia, nos artigos analisados, em termos de proporção, Ribeirão Preto, 1999

Os modelos psicodinâmico/psicanalítico e humanista/ centrado na pessoa são os mais freqüentemente citados. Segue-se o modelo fenomenológico e o menos citado é o modelo comportamental.

Quanto às estratégias metodológicas dos trabalhos empíricos, os resultados, em proporção, podem ser visualizados na Figura 5.

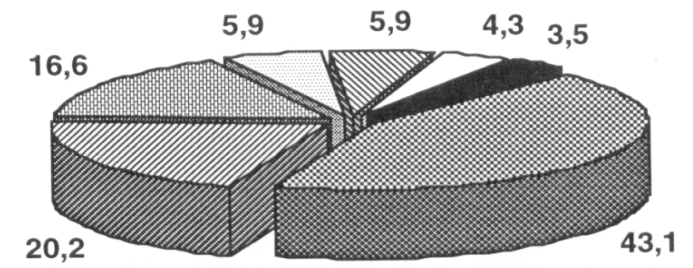

\begin{tabular}{|c|c|}
\hline $\begin{array}{l}\text { 图 entrevista } \\
\text { 国 observação } \\
\text { \&prontuários }\end{array}$ & $\begin{array}{l}\text { Q questionário } \\
\square \text { grupos } \\
\square \text { relato de atendimento }\end{array}$ \\
\hline
\end{tabular}

Figura 5 - Gráfico da estratégias metodológicas referidas no conjunto dos artigos analisados, em termos proporcionais, Ribeirão Preto, 1999

Verifica-se que a maior parte dos trabalhos utiliza a entrevista como recurso principal de coleta de dados. Seguem-se os questionários e observações. Quanto a esta última, nem sempre estava muito claro o modo como ela foi realizada. 0 trabalho em grupo apareceu, seja como estratégia de coleta de dados, seja como estratégia de intervenção. A análise de prontuários é outra forma de coleta de dados, seguido do relato de atendimentos. Na categoria outros, aparecem o emprego de testes psicológicos, escalas de avaliação de desenvolvimento, de atitude, análise de aula e uso de role playing.

\section{DISCUSSÃO}

Buscou-se, no presente trabalho, identificar como vem ocorrendo a inserção da Psicologia na Enfermagem, visto que a primeira vem sendo chamada a participar na construção do conhecimento sobre os processos envolvidos na saúde e doença.

Um primeiro aspecto diz respeito ao tema psicológico. Quando se afasta do modelo tradicional da Enfermagem, próximo ao modelo médico biológico, a visão dos fenômenos relacionados ao lidar com a saúde doença de sua clientela e mesmo com sua própria atuação profissional, requer a abordagem aos aspectos psicológicos (emocionais, comportamentais). É a busca de ver o ser humano, destinatário do trabalho do enfermeiro, de uma forma completa, abarcando as diferentes facetas de seu funcionamento.

0 uso de referência a obras de Psicologia é predominante nos artigos focalizados. Entretanto, o fato de boa parte dos trabalhos não utilizá-las, apesar de abordar aspectos psicológicos, revela que a Enfermagem já construiu um corpo de conhecimento próprio que prescinde dessa relação mais direta com trabalhos de Psicologia, no que tange à compreensão do doente e do adoecimento, à interação enfermeiro paciente/cliente e, ainda, quanto às reflexões sobre sua atuação profissional. Pode-se dizer que no terreno do psicológico, as duas áreas se fundem provocando "a aparente confusão que caracteriza as ciências humanas em nossos dias ${ }^{(10)}$, citando Foucault.

Quando se focaliza a participação de profissionais de outras áreas do conhecimento, em especial do psicólogo, na produção de trabalhos de Enfermagem verifica-se que ela ocorre, embora de modo pouco freqüente quer se trate de participação de docente não enfermeiro, quer se trate de outros profissionais não docentes e não enfermeiros.

Quanto às estratégias metodológicas, são empregados recursos de uso amplo nas Ciências Sociais/Humanas, sendo que as estratégias de entrevista e questionários são as mais freqüentemente utilizadas. Quando se quer saber o significado de uma situação para uma pessoa pode-se observá-la nessa situação ou perguntar a ela o que pensa ${ }^{(11)}$. De modo convergente com a tendência geral dos trabalhos analisados de adotarem como modelo teórico os referenciais psicodinâmicos, humanistas e fenomenológicos, parece mais razoável optar-se pela alternativa 
de falar com a pessoa. As estratégias de observação tem sido menos freqüentemente empregadas porque elas parecem estar ligadas a uma visão positivista do conhecimento e a uma "coisificação" do ser humano da qual, mais recentemente, se tem procurado afastar. A utilização da entrevista ${ }^{(12)}$ segue o sentido de se construir, com os sujeitos, uma compreensão da realidade focalizada.

Para superar uma limitação deste trabalho, relativa à gênese dos trabalhos publicados, sugere-se focalizar a produção gerada no contexto específico dos programas de pós-graduação, sejam aqueles da Enfermagem nos quais encontram-se como docentes orientadores, psicólogos, seja nos programas de Psicologia que recebem enfermeiros. Ao proceder à incursão pelos periódicos, aqui mencionados, verificou-se, não raras vezes, notas de rodapé

\section{REFERÊNCIAS BIBLIOGRÁFICAS}

1. Hall C, Lindsey G. Teorias da personalidade. São Paulo: EPU; 1973.

2. Botega N. 0 ensino da psicologia médica: uma enquete postal. Ver ABP-APAL 1994; 16(2): 45-51.

3. Christófaro MAC. Currículo mínimo para a formação do enfermeiro: na ordem do dia. Rev Bras Enfermagem 1991; 44(2/3): 7-9.

4. Manzolli MC. Formação do enfermeiro: contribuições da psicologia. São Paulo: Sarvier; 1985.

5. Birman J. Enfermidade e loucura. Rio de Janeiro: Editora Campus; 1980.

6. Ramos-Cerqueira MT. A interdisciplinaridade e psicologia na área da Saúde. Temas Psicol 1994; 3: 37-41. que informavam ser aquele artigo produto de dissertaç̃̃es ou teses.

Vale, ainda, comentar as diferenças verificadas neste trabalho e em outro ${ }^{(7)}$ quanto à proporção de trabalhos enquadrados na categoria estados psíquicos. Uma das razões das diferenças reside nos períodos focalizados pelos dois trabalhos, outra, no procedimento de categorização e uma última seria relativa à procedência dos mesmos.

Acredita-se que este tipo de trabalho seja útil não apenas por sugerir uma discussão sobre interdisciplinaridade e transdisciplinaridade, mas por fornecer bases para o planejamento do ensino de disciplinas de Psicologia, tanto para a Enfermagem como para outros cursos de formação de profissionais da área da Saúde.

7. Pereira LO, Inocenti A, Silva GB. A iniciação científica na graduação em enfermagem na USP (1993 a 1996): análise crítica. Rev Latinoam Enfermagem 1999; 7(3): 77-86.

8. Davidoff L. Introdução à psicologia. São Paulo: Makron Books do Brasil; 1986.

9. Todorov JC. A psicologia como o estudo das interações. Psicologia: teoria e pesquisa 1989; 5(2): 325-47.

10. Japiassu H. Introdução à epistemologia da psicologia. Rio de Janeiro: Imago; 1982.

11. Skinner BF. Recent issues in analysis of behavior. Columbus: Merril Publishing; 1989.

12. Gamboa SAS. A dialética na pesquisa em educação: elementos de contexto. In: Fazenda I, organizador. Metodologia da pesquisa educacional. São Paulo: Cortez; 1989.

Recebido em: 28.4.2000

Aprovado em: 8.12.2000 